\title{
Penfluridol targets acid sphingomyelinase to inhibit TNF signaling and is therapeutic against inflammatory autoimmune diseases
}

Yue-hong Chen ${ }^{1,2}$, Rong-han Liu' ${ }^{1}$, Ya-zhou Cui ${ }^{1}$, Aubryanna Hettinghouse' ${ }^{1}$ Wen-yu Fu' ${ }^{1}$ Lei Zhang ${ }^{1}$, Chen Zhang ${ }^{1}$ and Chuan-ju Liu' ${ }^{1,3^{*}}$

\begin{abstract}
Background: Penfluridol, isolated from an FDA-approved small-molecule drug library as an inhibitor of tumor necrosis factor a (TNFa)-stimulated NF-KB activation, is clinically used to treat chronic schizophrenia and related disorders. This study is aimed to investigate the therapeutic effect of penfluridol on TNFa-stimulated inflammatory autoimmune diseases, particularly inflammatory arthritis.

Methods: Various in vitro studies to confirm the inhibitory effect of penfluridol on TNFa-induced NF-KB activity in bone marrow-derived macrophages or Raw 264.7 macrophage cell line. In vivo studies assessed the therapeutic effects of penfluridol in various disease models, including TNFa transgenic mice, collagen-induced arthritis, DSSinduced colitis, and TNBS-induced colitis. Identification and characterization of the binding of penfluridol to acid sphingomyelinase using bioinformatics and drug affinity responsive target stability assay. Acid sphingomyelinase activity assays to reveal penfluridol-mediated inhibition of acid sphingomyelinase activity. siRNA knockdown experiments to illustrate the dependence of penfluridol's anti-TNF activity on acid sphingomyelinase.

Results: Penfluridol effectively inhibited TNFa-induced NF-KB activation in vitro and alleviated the severity of arthritis and colitis in vivo. Mechanistic studies revealed that penfluridol bound to acid sphingomyelinase and inhibited its activation. In addition, knockdown of acid sphingomyelinase largely abolished the inhibitory effects of penfluridol on TNFa-induced inflammatory cytokine production. Furthermore, penfluridol suppressed the differentiation of spleen naive CD4+T cells to $\mathrm{TH} 1$ and $\mathrm{TH} 17$ and inhibited $\mathrm{M} 1$ macrophage polarization.
\end{abstract}

Conclusion: This study provides the rationale for the possible innovative use of penfluridol as a newly identified small-molecule drug for TNFa-driven diseases, such as inflammatory arthritis and colitis.

Keywords: Acid sphingomyelinase, Arthritis, Colitis, Penfluridol, Tumor necrosis factor a, NF-KB

\section{Introduction}

Tumor necrosis factor alpha (TNF $\alpha)$, a pro-inflammatory cytokine released by several cell types such as macrophages and monocytes, plays an important role in

\footnotetext{
${ }^{*}$ Correspondence: chuanju.liu@nyumc.org

${ }^{1}$ Department of Orthopaedic Surgery, New York University Grossman

School of Medicine, Rm 1608, HJD, 301 East 17th Street, New York, NY 10003, USA

Full list of author information is available at the end of the article
}

normal inflammatory response and immune regulation. However, dysregulation of TNF $\alpha$-stimulated NF-kB signaling is implicated in wide ranges of diseases, including the autoimmune diseases rheumatoid arthritis (RA) and inflammatory bowel disease (IBD) [1, 2]. Correspondingly, blocking TNF $\alpha$ activity or TNF $\alpha$-stimulated NF- $\mathrm{kB}$ signaling is accepted as an effective treatment strategy [3]. Currently, there are five biologic TNF $\alpha$ inhibitors (TNFi; adalimumab, certolizumab pegol, etanercept, 
golilmumab, infliximab) which have been approved for clinical use, and the most common application is to treat RA and IBD [4]. Although effective for some patients, TNFi therapy fails to deliver an ACR50 response in up to $50 \%$ of RA patients, who need to switch to a second TNFi agent or other biologics [5, 6]. Moreover, adverse effects of TNFi occur in $15 \%$ of patients and drug regimens are associated with high cost, approximately $\$ 40,000$ per year for a patient [6-8].

Development of new drugs that are cheaper and have less adverse effects than TNFi is urgently needed to fill the non-effective treatment gap, though progress is impeded by the extensive cost of bringing a new drug to market, requiring 15 years and $\$ 800$ million on average [9]. Fortunately, quick screening of FDA-approved onmarket small-molecule drug library makes this possible. Through in vitro screening using NF-kB-bla THP-1 cell line and in vivo screening using human TNF $\alpha$ transgenic (hTNF-TG):NF-kB-Luc double mutant mice, penfluridol was identified as one of five small-molecule drugs that could inhibit TNF $\alpha$-stimulated NF-kB activation [10]. Clinically, penfluridol, a first generation diphenylbutylpiperidine antipsychotic with a long half-life of $66 \mathrm{~h}$, is a typical highly potent small-molecule drug used to treat chronic schizophrenia and related disorders [11-14], with treatment efficacy and risk of adverse events similar to chlorpromazine, a benchmark antipsychotic for schizophrenia. Furthermore, penfluridol has a high treatment compliance which benefits from its long half-life $[15,16]$. In addition to acting as a typical antipsychotic by targeting D2-like dopamine receptor, penfluridol is also a $\mathrm{Ca}^{2+}$-calmodulin inhibitor that inhibits hormonal secretion and decreases growth hormone in a dose-related manner [17]. Here, we report penfluridol is a promising drug for treatment of autoimmune diseases, particularly RA and IBD.

\section{Materials and methods \\ Mice}

Both 8-week-old male and female hTNF-TG mice (Taconic Biosciences), male 10-12 week-old DBA/J1 mice (Jackson Laboratory), and male 8-week-old wildtype (WT) C57BL/6 mice (Jackson Laboratory) were used for experiments. Mice, 5 in a cage, were housed in a rodent barrier facility at Skirball Animal Facility of New York University Langone Medical Center on a 12-h light-dark cycle with ad libitum access to food and water. Mice with weight 18-22 g that were in good health condition were enrolled for experiments and no mice were excluded. When starting experiments, animals were randomly assigned to each group on the basis of random number table, six mice were assigned to each group based on empirical choice. All animal experiments were performed in accordance with protocols approved by the Institutional Animal Care and use Committee of New York University School of Medicine.

\section{Reagents and materials}

Dulbecco's modification of Eagle's medium (DMEM, 10-017-CM) was purchased from Corning, and fetal bovine serum (FBS, S1620) was bought from Biowest. Human TNFa/TNFSF1A (NFTA0), IL-12 (419-ML010), TGF-beta (240-B-002) were bought from R\&D Systems. Penfluridol (PF, 26864-56-2) and methotrexate (59-05-2) were bought from MedChem Express. Type II chicken collagen (20012), complete Freund's adjuvant (7001), and incomplete Freund's adjuvant (7002) were purchased from Chondrex. Dextran sulphate sodium salt (DSS, molecular weight: $36000-50000 \mathrm{Da}$ ) was bought from MP Biomedicals. Picrylsulfonic acid solution (Synonym: 2,4,6-Trinitrobenzenesulfonic acid solution, TNBS, 92822), 5-aminosalicylic acid (5-ASA, A-3537), and LPS (L2630) were bought from Sigma-Aldrich. TransAM ${ }^{\circledR}$ NFkB p65 Activation Assay kit (40096) was bought from ACTIVE MOTIF. 3.5-French 38-cm catheter (4193505) was bought from Utah Medical Products, Inc. Plastic feeding tubes, 20 ga $\times 38 \mathrm{~mm}$ (FTP-20-3850) were bought from INSTECH Laboratories. Antibodies against p-Erk1/2 (4370s), t-Erk1/2 (4695), p-p38 (9211s), t-p38 (9212), p-JNK (9255s), t-JNK (9258), t-p65 (4764s), and GAPDH (2118) were purchased from Cell Signaling Technology. Receptor activator of nuclear factor kappa-B ligand (RANKL, sc-9073) and Lamin B (sc6216) were bought from Santa Cruz. RNeasy ${ }^{\circledR}$ Mini Kit (74106) and Random Hexamers (79236) were bought from Qiagen. TNF $\alpha$ (PHC3015), mouse IL-1 $\beta$ enzymelinked immunosorbent assay (ELISA) kit (88-7013), and mouse IL-6 ELISA kit (88706476) were purchased from Invitrogen. VECTASHIELD ${ }^{\circledR}$ Mounting medium with DAPI (H-1200) was bought from Vector Laboratories. SYBR $^{\circledR}$ Green PCR Master Mix (4309155) was bought from Applied Biosystems. ImProm-II ${ }^{\mathrm{TM}}$ Reverse Transcriptase (M314C) and Dual-Luciferase reporter assay system (E1910) were purchased from Promega Corporation. Nitrocellulose Membranes $(0.45 \mu \mathrm{m}, 162-0115)$ was bought from Bio-Rad. RIPA Lysis Buffer system (sc24948A) was bought from ChemCruz. Macrophage Colony-Stimulating Factor (M-CSF, 576406), IFN $\gamma$ (505702), IL-4 (504102), PE-CD25 (102008), anti-CD3 (100302), anti-CD28 (102102), anti-IL4 (504102), anti-IFNY (505702), and recombinant mouse IL-6 (575702) were bought from Biolegend. ACK Lysing Buffer (A10492-01) was bought from Gibco. Pronase from Streptomyces griseus (PRON-RO) was bought from Roche. Acid Sphingomyelinase Assay Kit (K-3200) was bought from echelon. Antibody against acid SMase/SMPD1 (LS-C334919) 
was bought from LSBio. Silencer ${ }^{\circledR}$ Select Negative Control siRNA (4390843) was bought from life technologies. TRIzol reagent (15596026), mouse SMPD1 siRNA (151776, AM16708), and NE-PER nuclear and cytoplasmic extraction reagents (78833) were bought from Thermo Fisher Scientific. Fixation/Permeabilization Solution Kit with BD GolgiPlug ${ }^{\mathrm{TM}}$ (555028) was bought from BD Biosciences. Mouse Naïve CD4+T cell Isolation Kit (130-104-453) and MACS Separation Columns were bought from Miltenyi Biotec. FITC-CD4 (M1004502) was bought from Sungene. Percp-cy5.5-IFNY (45-731182), PE-IL-17A (12-7177-81), and Alex-Fluo647-FoxP3 (51-5773-80) were bought from eBioscience. PE-IL4 (554389) was bought from BD Pharmingen.

\section{Bulk RNA-Seq}

BMDMs were treated with TNF $\alpha(10 \mathrm{ng} / \mathrm{mL})$ in the presence or absence of PF $(1 \mu \mathrm{M})$ for $24 \mathrm{~h}$. Cells were collected to extract total mRNA using RNeasy Plus Mini kit, and mRNA samples were sent to the Genome Technology Center at NYU Langone Medical Center for transcriptome sequencing. RNA-seq transcriptome library was prepared using $1 \mu \mathrm{g}$ of total RNA. Messenger RNA was isolated based on polyA selection method and then fragmented by fragmentation buffer. Double-stranded cDNA was synthesized and was subjected to end-repair, phosphorylation, and "A" base addition. Libraries were size selected for cDNA target fragments of about $300 \mathrm{bp}$ on 2\% Low Range Ultra Agarose followed by PCR amplified for 15 PCR cycles. After quantification by TBS380, paired-end RNA-seq sequencing library was sequenced with the Illumina HiSeq xten sequencer $(2 \times 150 \mathrm{bp}$ read length).

Those genes downregulated after TNF $\alpha$ stimulation and treatment with penfluridol were used to draw a heatmap and analyze the gene expression differences. We used the online tool TFactS (http://www.tfacts.org/) to identify the transcription factors that regulated those downregulated genes.

\section{NF-kB luciferase assay}

NF- $\mathrm{kB}$ luciferase assay was performed according to previously published methods [18]. 293T cells were seeded in 24-well plate and transfected with NF-кB luciferase reporter gene plasmids $(1.5 \mu \mathrm{g})$ and Renilla plasmids $(0.3$ $\mu \mathrm{g})$ by lipofectamine 2000 for $8 \mathrm{~h}$. PF was added at concentrations of $0.001,0.01,0.1,1.0$, and $2.5 \mu \mathrm{M}$ overnight. The next day, cells were stimulated with TNF $\alpha(10 \mathrm{ng} /$ $\mathrm{mL}$ ) for $4 \mathrm{~h}$. Measurements of luciferase activity were conducted based on the manufacturer's specifications. Briefly, $100-\mu \mathrm{L}$ luciferase assay reagents (LARII) were added to wells followed by $20-\mu \mathrm{L}$ cell lysates and mixture with a pipette tip. Firefly luciferase was measured at a wavelength of $560 \mathrm{~nm}$. After adding $100 \mu \mathrm{L}$ Stop \& Glo ${ }^{\circledR}$ Substrate, a second measurement for Renilla luciferase was performed at $480 \mathrm{~nm}$. The fluorescence results were standardized to Renilla luciferase.

\section{Western blotting}

When inducing BMDMs, freshly isolated cells from the bone marrow were seeded in 6 -well or $10-\mathrm{cm}$ plates. BMDMs in 6-well plates were subjected to reduced serum conditions with DMEM supplemented with $2 \%$ FBS overnight, followed by adding DMSO or penfluridol $(1 \mu \mathrm{M})$ for $2 \mathrm{~h}$ before stimulating with TNF $\alpha(10$ $\mathrm{ng} / \mathrm{mL}$ ) for 15,30 , or $60 \mathrm{~min}$. Protein samples were prepared by lysing cells in RIPA buffer containing PMSF, proteinase inhibitor cocktail, and $\mathrm{NaVO} 4$. After determining the protein concentrations by bicinchoninic acid (BCA) assay, the proteins were boiled for $5 \mathrm{~min}$ in SDS sample buffer. BMDMs in 10-well plates were maintained in DMEM with 2\% FBS overnight, followed by adding DMSO or penfluridol $(1 \mu \mathrm{M})$ for $2 \mathrm{~h}$ and then stimulated with TNF $\alpha(10 \mathrm{ng} / \mathrm{mL})$ for 15,30 , or $60 \mathrm{~min}$. Cytoplasmic extracts (CE) and nuclear extracts (NE) were prepared using a commercially available kit (NE-PER nuclear and cytoplasmic extraction reagents, 78833, Thermo Fisher Scientific). A total of $20 \mu \mathrm{g}$ protein samples was separated by gel electrophoresis and transferred to a nitrocellulose membrane using a wet transfer system. The membrane was blocked in 5\% (w/v) non-fat milk in TBST for half an hour at room temperature. Incubation with primary antibody was carried out overnight at $4{ }^{\circ} \mathrm{C}$ followed by three TBST washes and application of secondary antibody for $1 \mathrm{~h}$ at room temperature. The bands on the membrane were developed by enhanced chemiluminescent substrate and visualized by a gel scanner.

\section{NF-KB DNA-binding activity}

BMDMs were treated with PF $(1 \mu \mathrm{M})$ overnight, then stimulated with TNF $\alpha(10 \mathrm{ng} / \mathrm{mL})$ for $4 \mathrm{~h}$. Cells were lysed and NF-kB DNA-binding activity was tested according to the manufacturer's specifications (TransAM ${ }^{\circledR} \mathrm{NFkB}$ p65 Activation Assay kit, 40096, ACTIVE MOTIF). Briefly, $\mathrm{NF}-\mathrm{kB}$ transcription factor was captured by binding to a consensus sequence $5^{\prime}$-GGGACTTTCC-3' which was immobilized on a 96-well plate. Nuclear extract $(5 \mu \mathrm{g})$ was added to each well and incubated with anti-NF- $\mathrm{KB}$ p65 antibody, followed by HRP-conjugated secondary antibody incubation. The absorbance was read on a SpectraMax i3x plate reader at a wavelength of $450 \mathrm{~nm}$. Results were expressed as the fold changes of NF- $\mathrm{KB}$ DNA-binding activity relative to control cells. 


\section{ELISA}

IL- $1 \beta$ and IL- 6 levels in cell cultural supernatants or in sera from animal models were measured by mouse ELISA kits based on the manufacturer's instructions. Optical density was measured by SpectraMax i3x plate reader at a wavelength of $450 \mathrm{~nm}$, and concentrations were calculated according to the standard curve. Supernatants from cells were prepared by addition of TNF $\alpha(10 \mathrm{ng} / \mathrm{mL})$ with or without PF $(0.1 \mu \mathrm{M}$ or $1 \mu \mathrm{M})$ for $24 \mathrm{~h}$.

\section{Real-time quantitative PCR}

Total RNA was extracted from inflamed joints and colons by TRIzol according to the manufacturer's instructions. To prepare samples, $10-\mathrm{mg}$ tissues were cut into small pieces and put into a grinding tube, then $1 \mathrm{~mL}$ TRIzol was added. Grinding of the prepared samples was performed with a tissue homogenizer, a PowerLyzer 24 (Qiagen). Samples were subsequently centrifuged at $10,000 \mathrm{~g}$ for $5 \mathrm{~min}$ at room temperature, collecting the supernatant for mRNA extracting.

Total RNA in cells was extracted with RNeasy plus mini kit and cDNA was synthesized using SuperScript ${ }^{\circledR}$ Reverse Transcriptase. SYBR ${ }^{\circledR}$ Green PCR Master Mix was used to perform real-time quantitative PCR (qRT-PCR) on a StepOnePlus ${ }^{\mathrm{TM}}$ real-time PCR System (Applied Biosystems). The mRNA expression levels were calculated by $\triangle \Delta \mathrm{CT}$, and fold changes of target mRNA levels were normalized to GAPDH. The following specific sequences as SYBR primers were used for the target gene expansion: mouse IL-1 $\beta\left(5^{\prime}-3^{\prime}\right)$ F: AAT CTC ACA GCA GCA CAT CA, R: AAG GTG CTC ATG TCC TCA TC; mouse IL-6 (5'-3') F: TTC CAT CCA GTT GCC TTC TTG, R: AGG TCT GTT GGG AGT GGT ATC; mouse NOS2 $\left(5^{\prime}-3^{\prime}\right)$ F: TGT TAG AGA CAC TTC TGA GGC TC, R: ACT TTG GAT GGA TTT GAC TTT GAA G; mouse IL-17 (5'-3') F: GGG AAG TTG GAC CAC CAC AT, R: TTC TCC ACC CGG AAA GTG AA; mouse MCP-1 (5'-3') F: GTC CCT GTC ATG CTT CTG G, R: GCG TTA ACT GCA TCT GGC T; mouse CXCL10 $\left(5^{\prime}-3^{\prime}\right)$ F: CCA AGT GCT GCC GTC ATT TTC, R: GGC TCG CAG GGA TGA TTT CAA; mouse GAPDH $\left(5^{\prime}-3^{\prime}\right)$ F: AGA ACA TCA TCC CTG CAT CC, R: AGT TGC TGT TGA AGT CGC.

\section{Isolation of bone marrow-derived macrophage}

Bone marrow-derived macrophages (BMDMs) were prepared from WT C57BL/6 mice. Following sacrifice, femurs and tibias were collected and cleared of tissue using sterile gauze. Bones were cut to open the medullary cavity and then centrifuged at $13000 \mathrm{~g}$ for $90 \mathrm{~s}$ to collect bone marrow cells. Cells were seeded in DMEM containing M-CSF $(10 \mathrm{ng} / \mathrm{mL})$, medium was refreshed every third day over a 6-day culture period. Then BMDMs were ready for experiments after stimulation with M-CSF for 6 days.

\section{hTNFa transgenic (hTNF-TG) mouse model}

Eight-week-old hTNF-TG mice, which express a human TNF $\alpha$ gene on a C57BL/6 background and can spontaneously develop arthritis, were used to test the treatment effect of penfluridol on a TNF $\alpha$-dominant arthritis model [19-21]. Body weight as well as clinical and deformity scores were assessed weekly. Clinical scores were taken as the sums of the scores from digits, paws, wrists, and ankles, and the highest score for each mouse was 24 . The detailed scoring systems were as follows: $0=$ normal, 0.2 $=$ swollen joint, for 20 digits; $0=$ normal, $1=$ noticeably swollen, $2=$ severely swollen, for 4 paws; $0=$ normal, 1 $=$ noticeably swollen, $2=$ severely swollen, for 2 wrists; $0=$ normal, $2=$ noticeably swollen, $4=$ severely swollen with stiffness of ankle joint, for 2 ankles. Deformity was ranked on a 0 to 3 scale, corresponding to absence of deformity, mild deformity, moderate deformity, severe deformity, and ankylosis, with the total highest score equaling 12. Prior to administration of drugs, hTNF-TG mice were randomly classified into to three groups $(n=$ 6/group): vehicle group, methotrexate group [22] (MTX, $2 \mathrm{mg} / \mathrm{kg}$, a positive treatment control) and penfluridol group $(10 \mathrm{mg} / \mathrm{kg})$. MTX and penfluridol were dissolved in DMSO and stored at $-80^{\circ} \mathrm{C}$ (for up to 3 months) after aliquoting. For in vivo application, drugs were diluted by a solution of water:ethanol:2\% acetic acid at 8:3:1 (v/v), making the final volume of DMSO less than $5 \%$. Drugs were delivered via oral gavage once a day using flexible plastic feeding tubes. For preventative treatment, drugs were given and clinical assessment began on the day when mice were 8 weeks old. For therapeutic treatment, drugs were given and clinical assessment began on the day when the average score was 8 . At the end of treatment and observation, mice were sacrificed and sera, knees, and ankles were collected.

\section{Collagen-induced arthritis model}

DBA/1J male mice aged 10-12 weeks were used to establish collagen-induced arthritis (CIA) model [23, 24]. A $100 \mu \mathrm{L}$ emulsification of type II chicken collagen and complete Freund's adjuvant was injected intradermally by a $27-\mathrm{G}$ syringe $1.5-2.0 \mathrm{~cm}$ away from the tail base. On the 21th day after the first immunization, a $100-\mu \mathrm{L}$ booster of type II chicken collagen and incomplete Freund's adjuvant were intradermally given. Clinical scores for erythema and swelling were recorded every other day thereafter until the end of the experiment. The scores were assessed according to the following standards: $0=$ normal, $1=$ mild swelling involving ankle, wrist, or one digit, $2=$ mild swelling involving entire paw or more 
than two digits, $3=$ moderate swelling from the ankle/ wrist to entire foot/paw and all digits, $4=$ severe swelling or ankylosing deformity of the whole ankle/wrist, foot/paw and digits. The total highest score for a mouse was 16, and a higher score indicated greater severity. Mice were randomly allocated into three groups: vehicle group, MTX (2 mg/kg), and penfluridol (10 mg/kg), with six mice in each group. For prevention groups, daily oral gavage of drugs was initiated on the 18th day after the first immunization while for therapeutic treatment groups, drug delivery began when clinical score had reached an average of 5 in each group. Mice were sacrificed at experimental endpoints, and sera and ankles were collected.

\section{DSS-induced colitis model}

Prior to initiation of dextran sulphate sodium (DSS)induced colitis, 8-week-old C57BL/6 mice were randomly segregated into vehicle, 5 -ASA $(50 \mathrm{mg} / \mathrm{kg}$, serving as a positive control), PF $0.4 \mathrm{mg} / \mathrm{kg}$, PF $2 \mathrm{mg} / \mathrm{kg}$, and PF10 $\mathrm{mg} / \mathrm{kg}$ groups (6 mice in each group). Drugs were delivered via oral gavage once a day until sacrifice starting from 3 days before establishment of the DSS-induced colitis model via addition of 3\% DSS drinking water for 5 days and followed by normal drinking water for 3 days $[25,26]$. Scores of weight loss, stool consistency, and rectal bleeding were recorded every day beginning on the first day of 3\% DSS water supplementation and continuing until sacrifice. Disease activity index was the sum of the scores on weight loss, stool consistency, and rectal bleeding, the higher score correlating to more serious disease activity [27]. The detailed scoring systems were as follows: weight loss $(0=$ less than $5 \%, 1=$ between 5 and $10 \%, 2$ between 10 and $15 \%, 3=$ between 15 and $20 \%$, $4=$ over $20 \%)$, stool consistency $(0=$ normal, $2=$ loose stool, $4=$ diarrhea $)$, rectal bleeding $(0=$ negative, $2=$ blood trace, $4=$ gross blood). At the end of the experiment, mice were sacrificed, and sera and colons were collected. Colon length was measured by a caliper.

\section{TNBS-induced colitis model}

To establish 2,4,6-trinitrobenzenesulfonic acid (TNBS)induced colitis model [26], 8-week-old C57BL/6 mice were pre-sensitized by application of $150 \mu \mathrm{L} 1 \%$ TNBS on the depilated back between the two forelimbs. Five days later, the mice were randomly assigned to five groups: vehicle, 5-ASA ( $50 \mathrm{mg} / \mathrm{kg}$ ), penfluridol $0.4 \mathrm{mg} / \mathrm{kg}$, penfluridol $2 \mathrm{mg} / \mathrm{kg}$, and penfluridol $10 \mathrm{mg} / \mathrm{kg}$, six mice in each group. One week after pre-sensitization, the TNBSinduced colitis model was established by intrarectal injection of $100 \mu \mathrm{L} 3 \%$ TNBS. Drugs were supplied by oral gavage one time a day starting from the fifth day after pre-sensitization until the end of experiment. Body weight was recorded every day thereafter. Four days after modeling, mice were sacrificed and colons were collected.

\section{H\&E staining and quantitative analysis}

Fresh colons were fixed in $10 \%$ formaldehyde for $24 \mathrm{~h}$ before processed for paraffin embedding. Sections $(5 \mu \mathrm{m})$ were cut from tissue blocks and went through deparaffinized, rehydrated, routine $H \& E$ staining, dehydrated, and cleared for mounting.

H\&E-stained sections of ankle or knee joints were used to assess the severity of arthritis via scoring on the basis of inflammation, pannus formation, and cartilage damage [28]. The inflammation scores were 0,1 , and 2 corresponding to normal, local inflammatory cell infiltration, obvious inflammatory cell infiltration that formed lymphoid aggregates, and edema, respectively. Scores for pannus formation and cartilage damage were as follows: $0=$ normal, $1=$ pannus formation without articular cartilage infiltration, $2=$ pannus formation with articular cartilage infiltration.

H\&E staining score of colons [29]: the scores for colitis were the sum scores of inflammatory cell infiltration and intestinal wall structure integrity, the higher scores indicated more serious inflammation. For scores of inflammatory cell infiltration: $0=$ normal, $1=$ inflammatory cell only infiltrated to mucosa, $2=$ inflammatory cell infiltrated to mucosa and sub-mucosa, $3=$ inflammatory cell was found in the whole intestinal wall. Scores for intestinal wall structure integrity were assessed by the change of epithelial cell: $0=$ normal, $1=$ inflammatory cell was locally infiltrated, 2 = focally formed ulceration, $3=$ extensively formed ulceration with or without granulation tissue or pseudo-polyps.

\section{Prediction of penfluridol binding target}

Two steps were needed to predict the binding target of penfluridol. Firstly, finding out the canonical SMILES of penfluridol by visiting the online website, https://www. ncbi.nlm.nih.gov/pccompound. Secondly, visiting the prediction website (http://www.swisstargetprediction. $\mathrm{ch} /$ ) [30], an open website for drug target prediction, to find the possible binding target of penfluridol. The prediction result showed that the acid sphingomyelinase (ASM) was the target of penfluridol.

\section{DARTs}

Drug affinity responsive target stability (DARTs) experiments were performed to determine the binding between penfluridol and ASM based on previous reports [31, 32]. Cells were lysed by lysis buffer $(1 \times$ protease inhibitor cocktail, $50 \mathrm{mM}$ sodium fluoride, $10 \mathrm{mM}$ $\beta$-glycerophosphate, $5 \mathrm{mM}$ sodium pyrophosphate, 2 
mM sodium orthovanadate, $69 \%$ M-PER, V: V) on ice for $10 \mathrm{~min}$. Lysates were collected into a sterile pre-chilled $1.5-\mathrm{mL}$ tube and centrifuged at $18,000 \mathrm{~g}$ for $10 \mathrm{~min}$ at 4 ${ }^{\circ} \mathrm{C}$, and the supernatant was transferred to a new $1.5-\mathrm{mL}$ tube. After measuring the protein concentration by BCA assay, 99- $\mu \mathrm{L}$ cell lysates were mixed with $1 \mu \mathrm{L}$ DMSO or 1 $\mu \mathrm{L}$ penfluridol $(1 \mathrm{mM})$ and incubated with shaking for 30 min at room temperature. Cell lysate mixtures were then divided into $20-\mu \mathrm{L}$ aliquots for addition of pronase at a concentration of 1:800 or 1:1600 (pronase: total protein). Digestion was carried out for $5 \mathrm{~min}$ at room temperature and stopped by addition of $20 \times$ protease inhibitor cocktail and incubation on ice for $10 \mathrm{~min}$. Following digestion, samples were mixed with SDS-PAGE loading buffer and boiled for $5 \mathrm{~min}$ at $100{ }^{\circ} \mathrm{C}$. The samples were used to perform western blotting and ASM target bands were visualized by enhanced chemiluminescent substrate using a gel scanner.

\section{Acid sphingomyelinase activity}

Assays for ASM activity were performed according to the manufacturer's specifications. Briefly, Raw 264.7 cells at $70-80 \%$ confluence were treated with or without TNF $\alpha$ $(10 \mathrm{ng} / \mathrm{mL})$ overnight. After washing twice with phosphate-buffered saline, cells were collected into a $1.5-\mathrm{mL}$ tube in cell collection buffer by cell scraper. Then cells were lysed by freeze-thaw in liquid nitrogen for three cycles, and cell lysates were centrifuged at 14,000 rpm at $4^{\circ}$ for $10 \mathrm{~min}$. Supernatants were collected, and protein concentration was detected by BCA assay and was adjusted to $1.5 \mathrm{mg} / \mathrm{mL}$. In total, $20 \mu \mathrm{L}$ of cell lysates was added to a 96-well plate in the presence of varied concentrations $(0.1,1,10,100 \mu \mathrm{M})$ of penfluridol and incubated on a shaker for $1 \mathrm{~h}$ at room temperature. The following steps were on the basis of the manufacturer's specifications. Briefly, after incubating cell lysates with ASM substrate for $3 \mathrm{~h}$ at $37{ }^{\circ} \mathrm{C}$ and adding stop buffer, the plate was read at $360 \mathrm{ex} / 460 \mathrm{em}$. The activity of ASM in each group was calculated on the basis of standards.

\section{Spleen naïve $\mathrm{CD} 4+\mathrm{T}$ cell differentiation}

Induction of spleen naïve $\mathrm{CD} 4+\mathrm{T}$ cell differentiation into $\mathrm{T}$ cell subsets was performed according to previously reported methods [33, 34]. One day before cell differentiation induction, $1 \mathrm{~mL}$ PBS containing anti-CD3 (1 $\mu \mathrm{g} / \mathrm{mL})$ and anti-CD28 $(1 \mu \mathrm{g} / \mathrm{mL})$ was coated for TH1, TH2, and Treg and $1 \mathrm{~mL}$ PBS containing anti-CD3 (2 $\mu \mathrm{g} / \mathrm{mL})$ and anti-CD28 $(1 \mu \mathrm{g} / \mathrm{mL})$ was coated for TH17 in a 24-well plate and the plate was incubated at $4{ }^{\circ} \mathrm{C}$ overnight. The next day, spleens were isolated from WT C57BL/6 mice and spleen cells were isolated by grinding spleen on a $70-\mu \mathrm{m}$ mesh and collected in PBS containing $1 \%$ FBS, then centrifuged at $1000 \mathrm{rmp}$ for $5 \mathrm{~min}$ twice, followed by lysing red blood cells with ACK lysing buffer for 5 min on ice. After centrifugation, naïve CD4+T cells were isolated by $\mathrm{CD} 4+\mathrm{T}$ cell isolation kit in MACS separation columns according to the manufacturer's specifications. Coating buffer was aspirated, and the plate was washed once with PBS prior to seeding CD4+T cells $(1 \times$ $10^{6}$ per well) in $1.0 \mathrm{~mL}$ DMEM. The following cytokines were added to induce T cell differentiation: IL-2 (20 ng/ $\mathrm{mL})$, IL-12 (15 ng/mL), and anti-IL4 $(5 \mu \mathrm{g} / \mathrm{mL})$ for TH1; IL-2 $(20 \mathrm{ng} / \mathrm{mL}), \mathrm{IL}-4(10 \mathrm{ng} / \mathrm{mL})$, and anti-IFN $\gamma(5 \mu \mathrm{g} /$ $\mathrm{mL}$ ) for TH2; IL-6 (20 ng/mL), TGF $\beta$ (3 ng/mL), anti-IFN $\gamma(5 \mu \mathrm{g} / \mathrm{mL})$, and anti-IL4 $(5 \mu \mathrm{g} / \mathrm{mL})$ for TH17; IL-2 (20 $\mathrm{ng} / \mathrm{mL}), \operatorname{TGF} \beta(15 \mathrm{ng} / \mathrm{mL})$, anti-IFN $\gamma(5 \mu \mathrm{g} / \mathrm{mL})$, and anti-IL4 $(5 \mu \mathrm{g} / \mathrm{mL})$ for Treg. At the same time, penfluridol $(0.1 \mu \mathrm{M})$ or penfluridol $(1 \mu \mathrm{M})$ were added. Four days later, cells were collected for flow cytometry analysis.

To test the differentiation, $1 \mu \mathrm{L}$ Golgi stop buffer was added to each well for $4 \mathrm{~h}$. T cell subset stain was based on the specfications of Fixation/Permeabilization Solution Kit. TH1 were stained with FITC-CD4 and Percpcy5.5-IFN $\gamma$, TH2 were stained with FITC-CD4, PE-IL4, TH17 were stained with FITC-CD4 and PE-IL-17A, and Treg were stained with FITC-CD4, PE-CD25, and Alex-Fluo647-FoxP3. For intracellular staining, antibodies were diluted at 1:100 while, for cell surface staining, antibodies were diluted at 1:400. All samples were sent to NYU Langone Medical Center for detecting.

\section{Statistical analysis}

Assessment of clinical signs, colon length measurement, and histology scoring were performed by a pair of two investigators. Blinding was applied to one of the two investigators for the whole data collection process but not for data analysis. All data were organized and analyzed in GraphPad 8.0 and SPSS 22.0 statistics software. Data were expressed as mean with standard error of the mean. Statistical significance of the differences among groups was determined by one-way analysis of variance and Bonferroni post hoc test. The statistical differences of clinical scores among different groups were analyzed by repeated measures analysis of variance. $p<0.05$ was considered as statistically significant.

\section{Results}

\section{Penfluridol is isolated as an anti-NF-KB activation drug}

Through first screening a FDA-approved small-molecule drug library containing 1046 drugs using NF-кBbla THP1, secondary confirmation by NF-kB luciferase reporter assay, and third in vivo verification in hTNF-TG/ NF- $\kappa B$ luc mutant mice, we identified penfluridol as one of five drugs that could inhibit TNF $\alpha$-induced NF- $\mathrm{KB}$ activity [10]. Penfluridol is clinically used as an antipsychotic drug to treat various types of schizophrenia and 
A<smiles>OC1(c2ccc(Cl)c(C(F)(F)F)c2)CCN(CCCC(c2ccc(F)cc2)c2ccc(F)cc2)CC1</smiles>

C

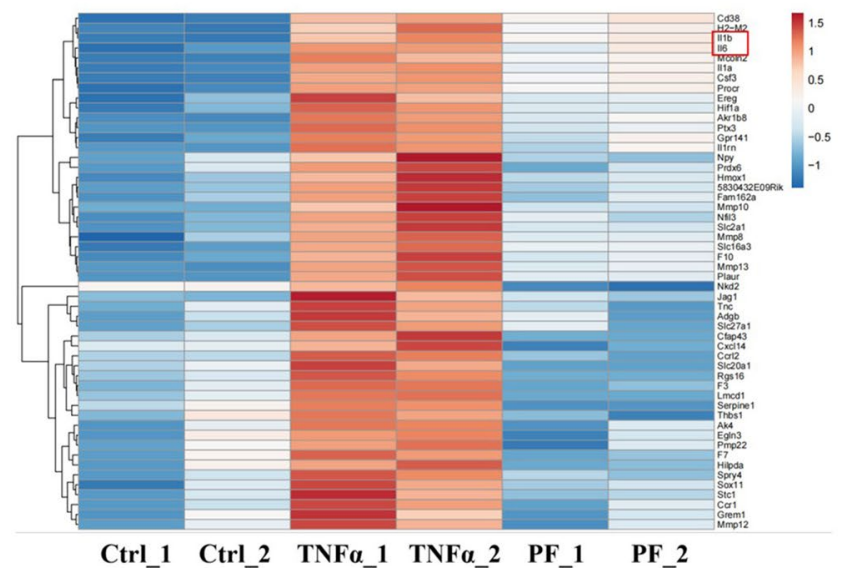

B

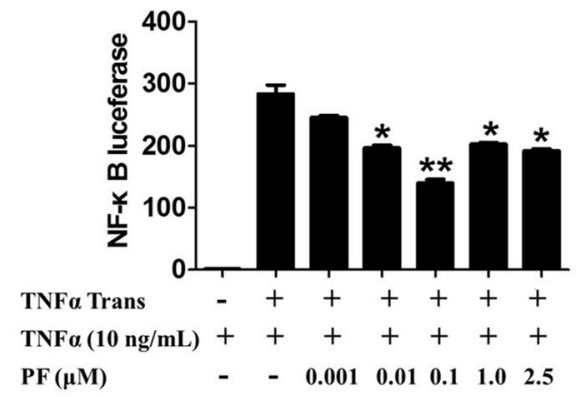

D

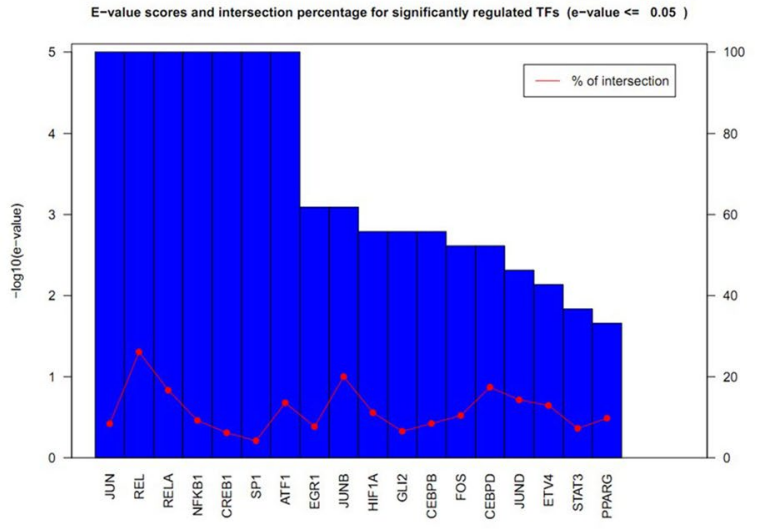

Fig. 1 Penfluridol is isolated as an anti-NF-KB activation drug. Penfluridol was identified as an anti-NF-KB activation drug stimulated by TNFa from a FDA-approved small-molecule drug library. NF-KB luciferase assay and bulk RNA-seq were used to confirm the inhibitory effect of penfluridol on TNFa-induced NF-kB activity. A The molecular structure of penfluridol (PF). B NF-kB luciferase assay of penfluridol at different doses in 293T. C Heat map analysis of down expression genes regulated by penfluridol after TNFa stimulation $(n=2)$. Penfluridol decreases a wide range of TNFa-induced gene expressions, including IL-1 $\beta$ and IL-6 indicated by a red rectangle. D Transcription factor enrichment analysis of RNA-Seq results. Penfluridol regulates a wide range of transcription factors, including RelA P65 and NF-KB1 P105. Experiments were performed for 3 biological replications, and one-way analysis of variance and Bonferroni post hoc test were used to test statistical significance of the differences among groups $\left({ }^{*} p<0.05,{ }^{* *} p<\right.$ 0.01). PF: penfluridol

its molecular structure is presented (Fig. 1A). To further confirm the inhibitory effect of penfluridol on TNF $\alpha$ induced NF- $\mathrm{kB}$ activity, NF- $\mathrm{kB}$ luciferase assay was performed in the presence of varied concentrations of penfluridol and results illustrated that penfluridol dosedependently reduced TNF $\alpha$-stimulated NF- $\mathrm{BB}$ activity (Fig. 1B). We also used bulk RNA-Seq to verify the inhibitory effect of penfluridol on TNF signaling downstream gene expressions after the activation of NF- $\mathrm{kB}$ by TNF $\alpha$. RNA-Seq results showed penfluridol decreased expressions of about 50 genes induced by TNF $\alpha$, including chemokine family members (such as CCR1, CCR12, CXCL14), interleukins (such as IL-1 $\alpha$, IL-1 $\beta$, IL-6), and matrix metalloproteinases (such as MMP8, MMP10, MMP12, MMP13), which are organized in the heatmap (Fig. 1C) and listed in the supplemental Table 1. We used the online tool TFactS (http://www.tfacts.org/) to identify the transcription factors that regulated these downregulated genes and found transcription factors RelA
P65 and NF-kB1 P105 were involved in the regulation of these genes which could be obviously regulated by penfluridol (Fig. 1D).

\section{Penfluridol inhibits TNFa-induced NF-KB activity in vitro}

To further verify the inhibitory effect of penfluridol on TNF $\alpha$-stimulated NF- $\mathrm{kB}$ activation in vitro, we assessed the phosphorylation of several molecules in the NF- $\mathrm{KB}$ signaling pathway, translocation of $\mathrm{p} 65$, binding activity of NF- $\mathrm{kB}$ p65 to DNA, and mRNA expression and release levels of several inflammatory cytokines in BMDMs or Raw 264.7 cells. When stimulating BMDMs with TNF $\alpha(10 \mathrm{ng} / \mathrm{mL})$, the phosphorylation levels of ERK, JNK, and p38 were significantly increased which were obviously inhibited by penfluridol $(1 \mu \mathrm{M})$ (Fig. 2A, Sfig. 1A-C). When stimulating BMDMs with TNFa (10 ng/mL), p65 translocated from cytoplasm to nuclei, which was inhibited by penfluridol $(1 \mu \mathrm{M})$ (Fig. 2B, Sfig. 1D-E). In addition, 


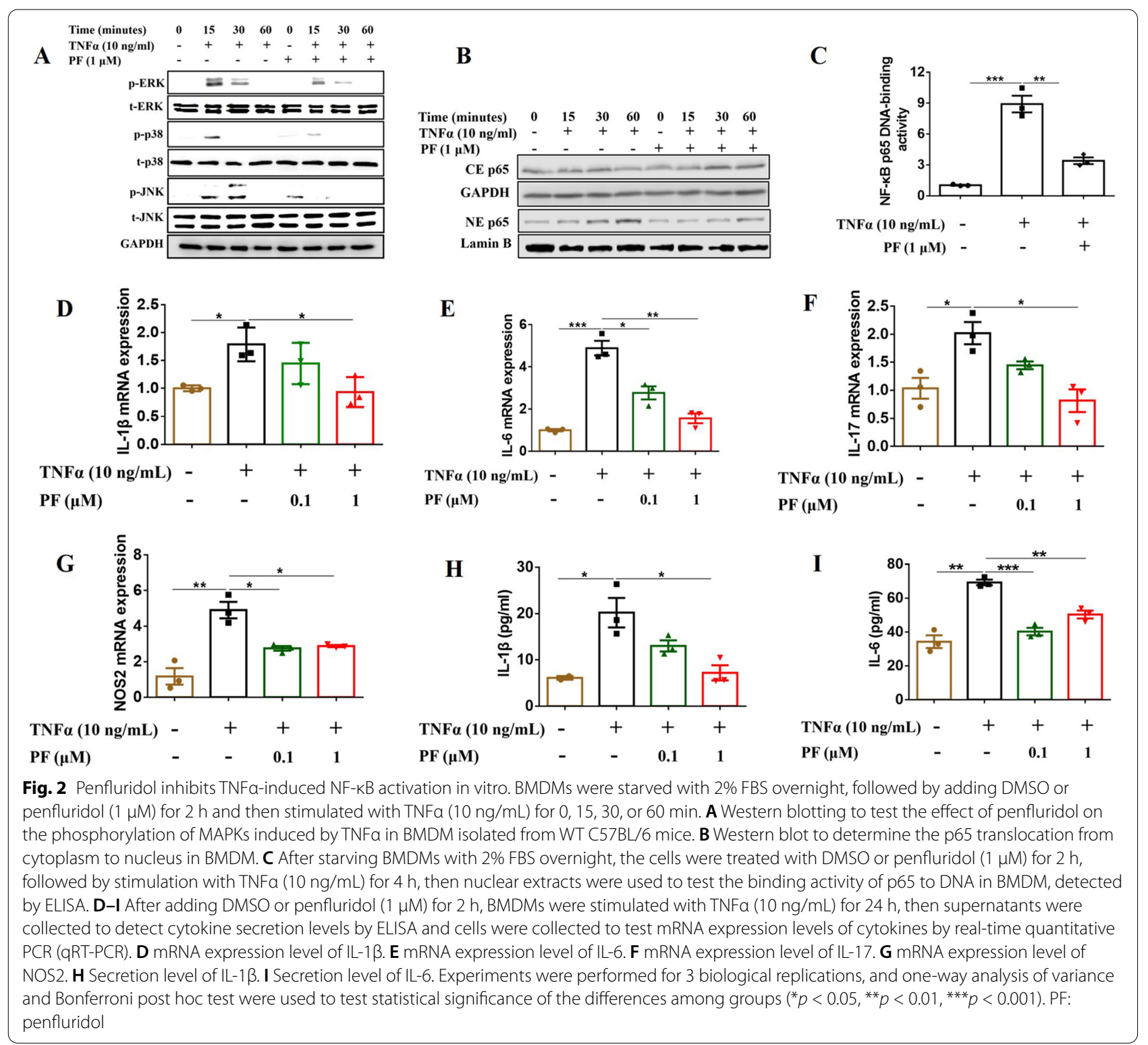

penfluridol reduced TNF $\alpha$-induced binding activity of p65 to DNA (Fig. 2C). qRT-PCR and ELISA assays further showed that penfluridol inhibited TNFo-induced mRNA expressions of IL-1 $\beta$, IL- 6 , IL-17, and NOS2 (Fig. 2D-G) and suppressed the cytokine secretion levels of IL-1 $\beta$ and IL-6 (Fig. $2 \mathrm{H}, \mathrm{I}$ ) in BMDMs isolated from WT C57BL/6 mice. The inhibitory effects of penfluridol on TNF $\alpha$-induced mRNA expression and secretion of inflammatory cytokines were also observed in macrophage cell line Raw 264.7 cells (Sfig. 2 A-F) and BMDMs isolated from hTNF-TG mice (Sfig. 3 A-E).

As NF- $\mathrm{KB}$ activity stimulated by TNF $\alpha$ also plays a pivotal role in osteoclastogenesis, the effect of penfluridol on osteoclastogenesis was examined and results revealed that penfluridol could markedly inhibit TNF $\alpha$ enhanced osteoclastogenesis (Sfig. 4 A-B). Penfluridol inhibited the production of inflammatory cytokines, such as TNF $\alpha, I L-1 \beta$, and IL-6, which are involved in regulating macrophage polarization [35]; accordingly, we also investigated whether penfluridol affected macrophage polarization. M1 macrophages were pro-inflammatory macrophages and secreted a wide range of inflammatory cytokines such as TNF $\alpha$, IL- $1 \beta$, and IL- 6 while M2 macrophages are anti-inflammatory macrophages, releasing anti-inflammatory cytokines like IL-10. Therefore, IL-6 and NOS2 were chosen as the markers for M1 macrophages while Arg1 and Mgl1 as the markers for M2 macrophages. The results suggested that penfluridol 


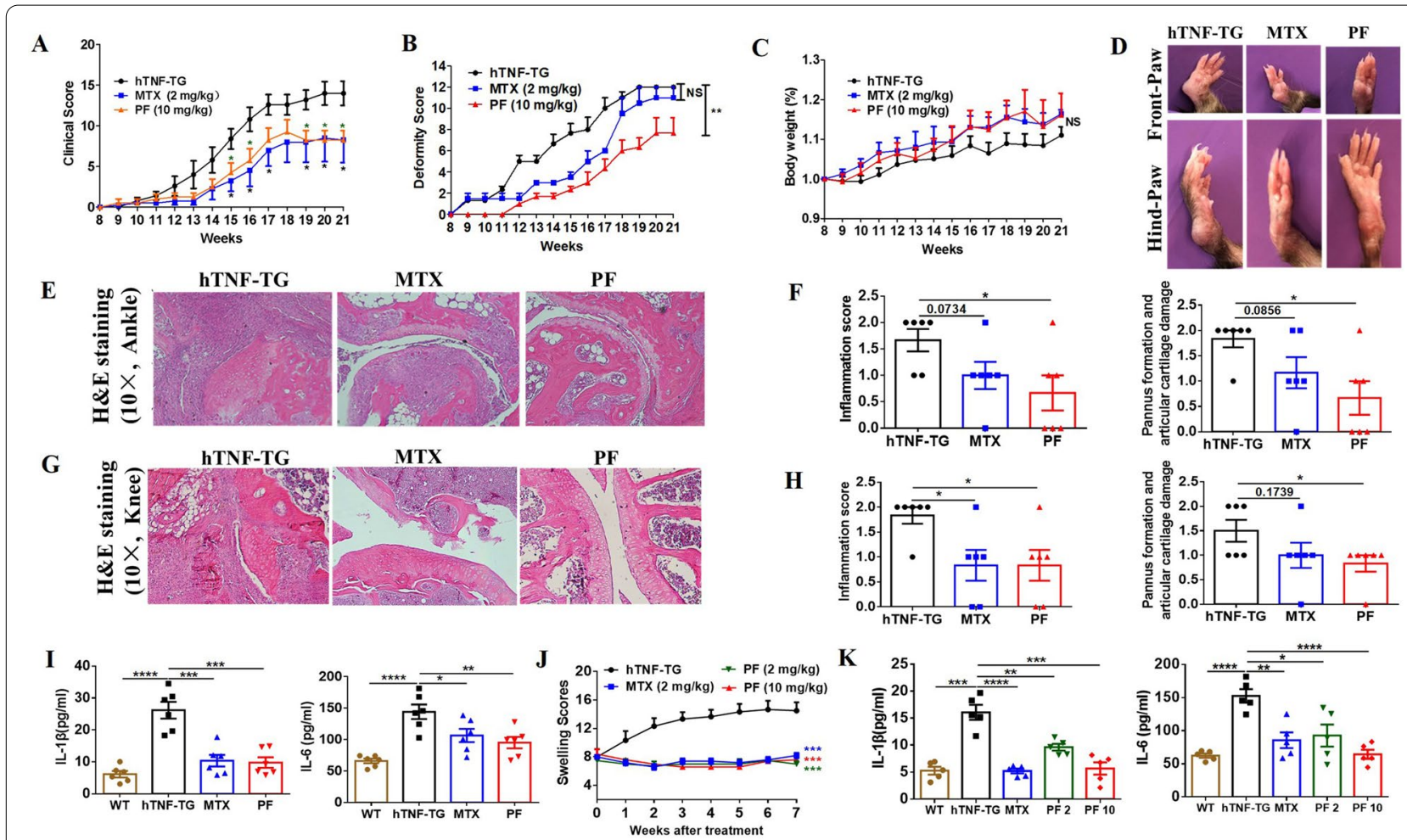

Fig. 3 Penfluridol prevents the onset and severity of arthritis in hTNF-TG mice. TNFa overexpression mouse model (hTNF-TG) which can spontaneously develop arthritis were used to observe the inhibitory effect of penfluridol on TNFa-induced NF-kB activation in vivo. Drugs were administrated by oral gavage once a day. Prevention treatment is penfluridol was given to mice at the eighth week of age when arthritis symptom began its onset; treatment of penfluridol was administrated when average clinical arthritis scores reached 8 points in each group. A Clinical score of prevention treatment. B Deformity score of prevention treatment. C Body weight of prevention treatment. D Representative pictures of both front and hind paws in each prevention treatment groups. E H\&E staining of paraffin-embedded ankle slides in prevention treatment groups. $\mathbf{F}$ Quantitative analysis of inflammation score and pannus formation and cartilage damage score of E. G H\&E staining of paraffin-embedded knee sections in preventive treatment groups. $\mathbf{H}$ Quantitative analysis of inflammation score and pannus formation and cartilage damage score of $\mathbf{G}$. I Serum IL-1 $\beta$ and IL-6 levels in preventive treatment groups, detected by ELISA. J Clinical scores in treatment groups. K Serum levels IL-1 $\beta$ and IL-6 in treatment groups, detected by ELISA. Six mice in each group. One-way analysis of variance and Bonferroni post hoc test were used to test statistical significance of the differences among groups, and repeated measures analysis of variance was used to test statistical differences of clinical scores among different groups $\left({ }^{*} p<0.05,{ }^{* *} p<0.01,{ }^{* * *} p<0.001,{ }^{* * *} p<0.0001\right)$. PF: penfluridol

inhibited M1 macrophage polarization evidenced by decreased mRNA expression levels of IL-6 and NOS2, but had no effect on M2 macrophage polarization evidenced by no alteration in M2 markers, Arg1 and Mg11 (Sfig. 4 C-F).

\section{Penfluridol prevents the onset and severity of arthritis in hTNF-TG mice}

The inhibitory effect on TNF $\alpha$-induced NF- $\mathrm{KB}$ activation was confirmed in vitro, which prompted us to investigate the treatment effect of penfluridol in vivo in TNF $\alpha$-dominant disease. hTNF-TG mice overexpressing human TNF $\alpha$ and spontaneously developing arthritis were used to test the treatment effect of penfluridol. Arthritis onset began approximately at 8 weeks of age with severity increasing longitudinally. Preventative treatment with penfluridol began at the 8 week of age to prevent progression of arthritis and weekly assessment of clinical signs coincided with commencement treatment. MTX served as the positive control as it is a smallmolecule drug that is the first line treatment for RA in clinic. Results showed that penfluridol could delay arthritis onset time and alleviate arthritis severity, with similar efficacy as the positive control drug MTX (Fig. 3A). As for the joint deformity, the treatment effect of penfluridol was superior to MTX, as demonstrated by the fact penfluridol could delay the deformity onset time and alleviate severity of joint deformity (Fig. 3B, D). Moreover, penfluridol did not affect body weight (Fig. 3C). $H \& E$ staining of ankle and knee sections suggested penfluridol reduced inflammatory cell infiltration, inhibited pannus formation, and protected cartilage from damage (Fig. 3E-H). To determine whether the reduced inflammatory cell infiltration and alleviated severity of joint 


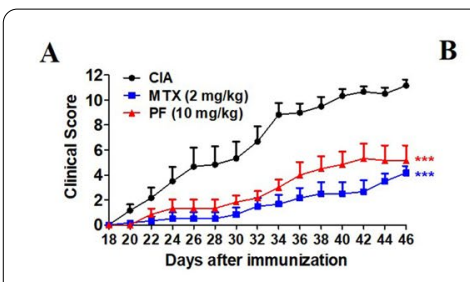

D

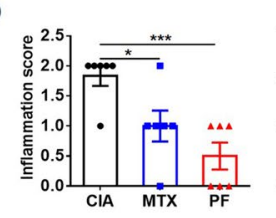

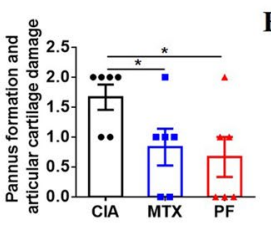

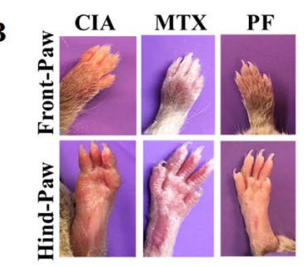

E

t.
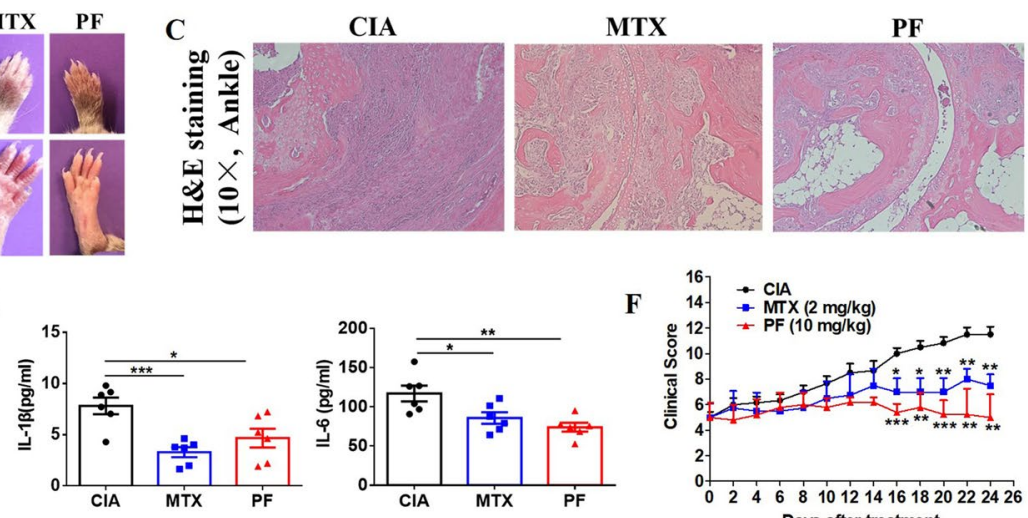

Fig. 4 Penfluridol alleviates severity of collagen-induced arthritis. Type II chicken collagen-induced arthritis (CIA) model were adopted to assess the prevention and treatment effect of penfluridol in NF-KB-activated arthritis and drugs were given one time a day by oral gavage. Preventive treatment was administrating penfluridol starting from the 18th day after the first immunization while therapeutic treatment was administrating penfluridol when clinical score reached an average of 5 in each group. A Clinical score in preventive treatment. B Representative pictures of both front and hind paws in preventive treatment groups. $\mathbf{C H} \& \mathrm{E}$ staining of paraffin-embedded ankle slides in preventive treatment groups. D Quantitative analysis of inflammation score and pannus formation and cartilage damage score of $\mathbf{C}$. E Serum levels of IL-1 $\beta$ and IL-6 levels in preventive treatment groups, detected by ELISA. F Clinical scores in treatment groups. Six mice in each group. One-way analysis of variance and Bonferroni post hoc test were used to test statistical significance of the differences among groups $\left({ }^{*} p<0.05,{ }^{* *} p<0.01,{ }^{* * *} p<0.001\right)$. PF: penfluridol

inflammation were attributable to the inhibitory effect of penfluridol on TNF $\alpha$-stimulated NF- $\mathrm{BB}$ activation, we tested downstream cytokines of NF- $\mathrm{kB}$ by qRT-PCR and ELISA. mRNA expression levels of chemokines CXCL10 and MCP-1 (Sfig. 5 A-B) in the inflamed joints and the production and release levels of cytokines IL- $1 \beta$ and IL- 6 in the sera were significantly reduced after treatment of penflurdol (Fig. 3I). In sum, penfluridol had a prophylactic effect in the TNF $\alpha$-dominant arthritis.

Further, we tested whether penfluridol had treatment effect to prevent disease progression in the TNF $\alpha$ dominant arthritis. Penfluridol was administered in the hTNF-TG mice when average clinical arthritis scores reached 8 points in each group. Results showed penfluridol could prevent disease progression (Fig. 3J) and reduce the production of inflammatory cytokines L- $1 \beta$ and IL- 6 in a dose-dependent manner (Fig. 3K). Collectively, penfluridol was effective in treatment of TNF $\alpha$-dominant arthritis.

\section{Penfluridol alleviates the severity of collagen-induced arthritis}

The effective preventive and therapeutic effects of penfluridol in TNF $\alpha$-dominant mouse model prompted us to test whether penfluridol had the same therapeutic effect in the diseases in which pathogenesis was dominant by TNF $\alpha$ and TNF $\alpha$-stimulated NF- $\mathrm{KB}$ activation, such as RA and IBD [2]. CIA mouse model, the most commonly used mouse model to imitate the pathogenesis of RA, was used to test the treatment effect of penfluridol in NF- $\mathrm{kB}$-activated arthritis. In our preventative treatment scheme, penfluridol was administrated starting from the 18th day after the first immunization before the disease onset. In a separate therapeutic treatment scheme, penfluridol was administrated when clinical score had reached an average of 5 in each group. Results of preventative treatment demonstrated penfluridol was associated with significantly reduced severity of arthritis, which was comparable to the firstline treatment MTX (Fig. 4A,B). The H\&E staining of ankles suggested that penfluridol inhibited inflammatory cell infiltration, suppressed pannus formation, and protected articular cartilage from damage (Fig. 4C,D). To further characterize the inhibitory effect of penfluridol on NF- $\mathrm{BB}$ activation, downstream cytokines of NF- $\mathrm{kB}$ were detected by qRT-PCR and ELISA. Results showed mRNA expressions of CXCL10 and MCP-1 were obviously decreased in inflamed joints (Sfig. 5 C-D) and production levels of inflammatory cytokines IL- $1 \beta$ and IL- 6 were statistically reduced in sera after penfluridol treatment (Fig. 4E). Results of therapeutic effects suggested penfluridol was associated with significantly reduced clinical scores, which was comparable to MTX treatment (Fig. 4F). In short, penfluridol was effective to prevent and treat CIA.

\section{Penfluridol is therapeutic against IBD mouse models}

As penfluridol could inhibit TNF $\alpha$-induced NF- $k B$ activity and was effective to alleviate the severity of 
A

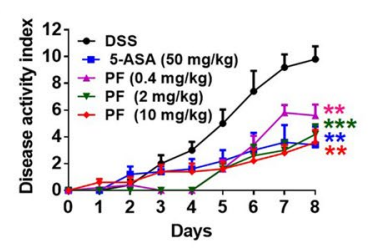

B

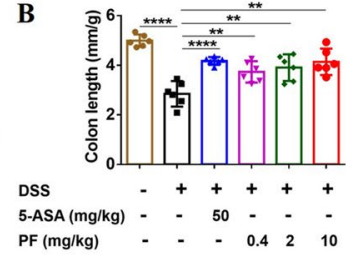

C

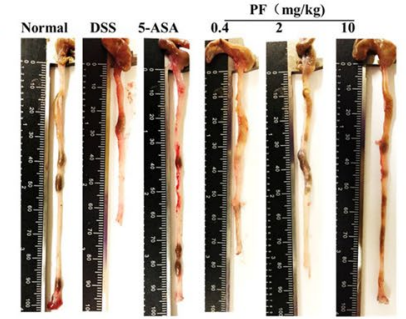

F
D

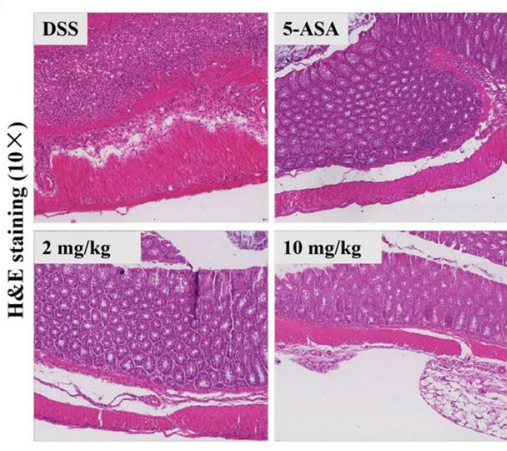

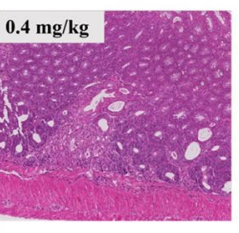

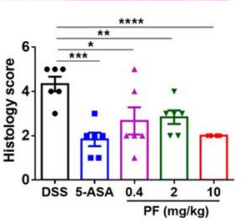

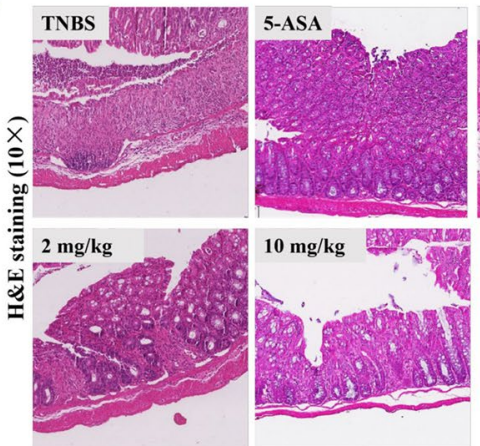

E
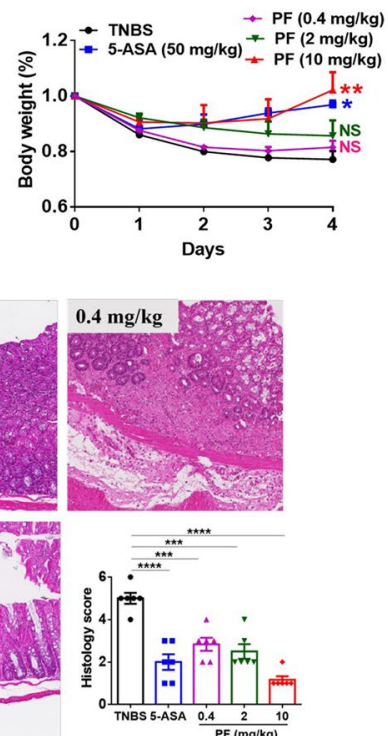

Fig. 5 Penfluridol is therapeutic against IBD mouse models. DSS-induced colitis and TNBS-induced colitis model were established to assess the treatment effect of penfluridol in NF-KB-activated colitis in which drugs were given once a day by oral gavage. A Disease activity index of DSS-induced colitis model. B Colon length of DSS-induced colitis model. C Representative colon length in each group of DSS-induced colitis model. D H\&E staining of colon tissues and quantitative analysis of H\&E staining of DSS-induced colitis model. $\mathbf{E}$ Body weight of TNBS-induced colitis model. F H\&E staining of colon tissues and quantitative analysis of H\&E staining of TNBS-induced colitis model. Six mice in each group. One-way analysis of variance and Bonferroni post hoc test were used to test statistical significance of the differences among groups, and repeated measures analysis of variance was used to test statistical differences of clinical scores among different groups (NS $=$ not significance, ${ }^{*} p<0.05,{ }^{* *} p<0.01,{ }^{* * *} p$ $\left.<0.001,{ }^{* * * *} p<0.0001\right)$. PF: penfluridol

TNF $\alpha$ and TNF $\alpha$-stimulated NF- $\mathrm{kB}$ activation-driven arthritis, we assessed the treatment efficacy of penfluridol in another TNF $\alpha$-dominant autoimmune disease, IBD. The DSS-induced colitis mouse model is a model which mimics ulcerative colitis (UC). Penfluridol treatment was initiated 3 days ahead of model establishment and 5-ASA was chosen as the positive control considering it is a small-molecule drug and is used as a firstline treatment. Penfluridol, at three doses, statistically reduced disease activity index to a degree comparable with the positive control drug 5-ASA (Fig. 5A) and was found to be effective in preventing shortening of colon length, the feature of UC (Fig. 5B,C). H\&E staining of the colons showed penfluridol was as effective as positive control drug 5-ASA to inhibit inflammatory cell infiltration and protect the integrity of the intestinal wall (Fig. 5D). The downstream molecules of NF- $\mathrm{kB}$ activity were detected by qRT-PCR and results showed mRNA expression levels of CXCL10 and MCP-1 were decreased in penfluridol-treated colon tissues (Sfig. 5 E-F).

We also tested the treatment effect of penfluridol in the TNBS-induced colitis mouse model, which resembles human Crohn's disease (CD). Penfluridol was administrated 5 days later after pre-sensitized of $1 \%$ TNBS. High-dose $(10 \mathrm{mg} / \mathrm{kg})$ penfluridol prevented body weight loss and was as effective as positive control drug 5-ASA while lower doses of penfluridol did not appreciably prevent body weight loss (Fig. 5E). H\&E staining of colons revealed that each tested dose of penfluridol was effective to inhibit inflammatory cell infiltration and protect the integrity of intestinal wall (Fig. 5F). The downstream molecules of NF- $\mathrm{kB}$ activity were detected by qRT-PCR, and results reported mRNA expression levels of CXCL10 and MCP-1 were obviously decreased in penfluridol-treated colon tissues (Sfig. 5 G-H). Collectively, penfluridol was therapeutic against IBD including UC and $\mathrm{CD}$.

\section{Acid sphigomyelinase is a target of penfluridol}

The inhibitory effect of penfluridol on TNF $\alpha$-induced NF- $\mathrm{KB}$ activation and anti-inflammation effects promoted us to explore the molecular mechanisms underlying penfluridol's anti-TNF $\alpha$ activity. For this purpose, we first examined whether penfluridol affected the binding between TNF $\alpha$ and TNF $\alpha$ receptors. We thus performed solid phase binding and flow cytometry and found that penfluridol did not impact the binding between TNF $\alpha$ 
A

Pronase: protein
PF $(100 \mu \mathrm{M})$

ASM

GAPDH

C

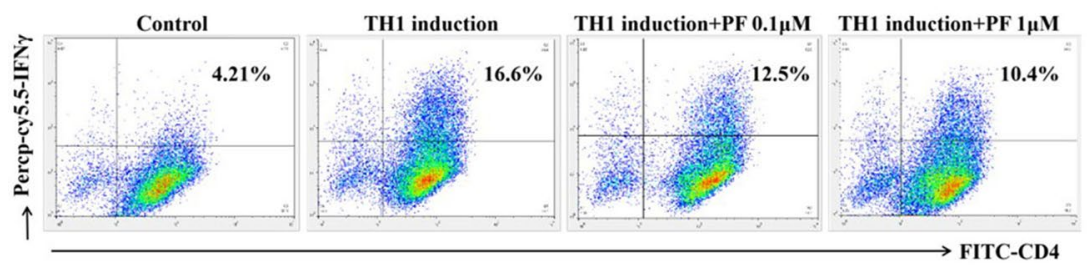

$\mathbf{E}$

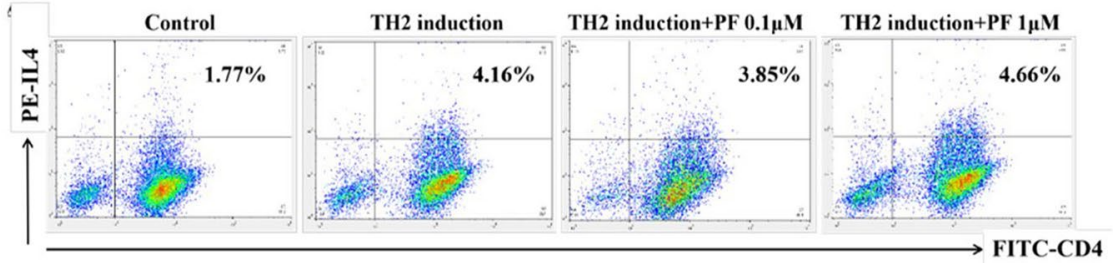

G

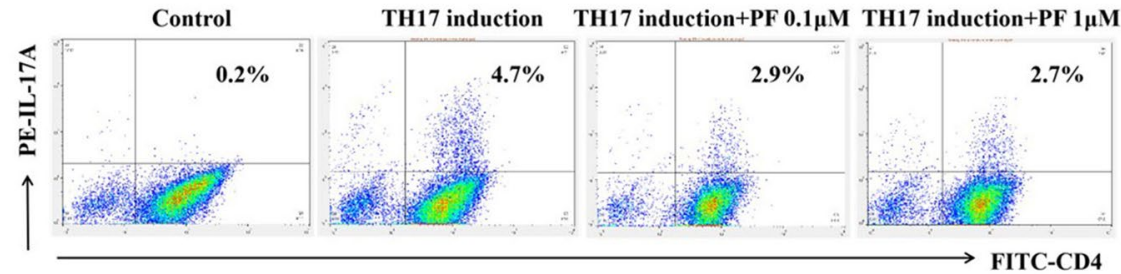

\section{I}

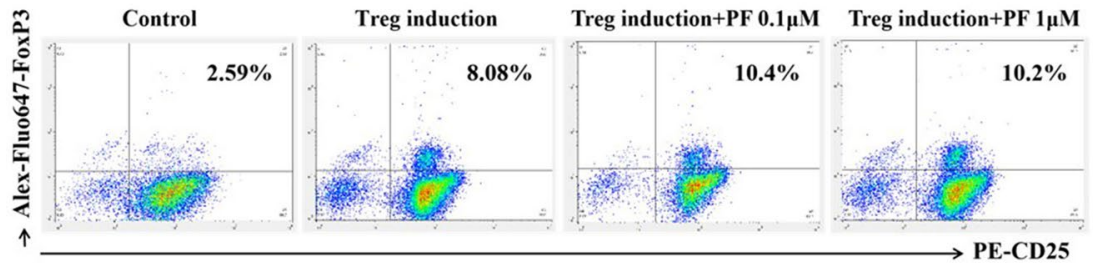

B

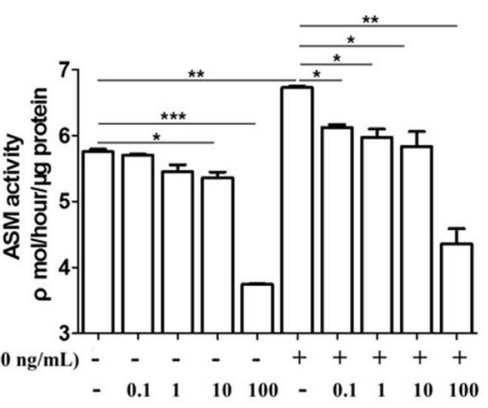

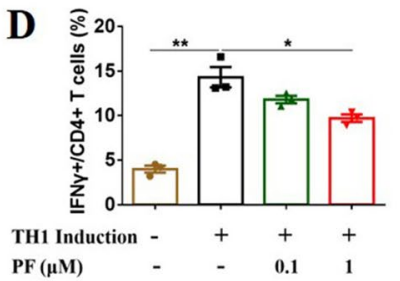
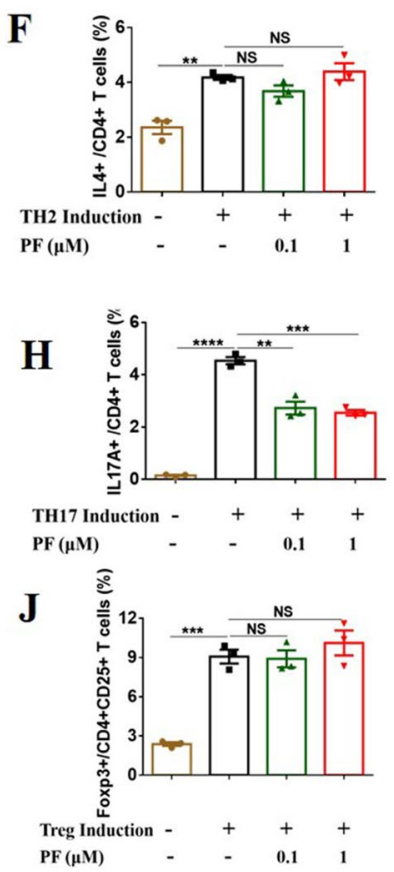

Fig. 6 Acid sphigomyelinase is a target of penfluridol. A Raw 264.7 cells were collected to perform DARTS assay. B Raw 264.7 cells were used to conducted ASM activity assay. Cells were treated in presence or absence of TNFa $(10 \mathrm{ng} / \mathrm{mL})$ overnight, then lysed by freeze-thaw in liquid nitrogen for three cycles, followed by collecting supernatants after centrifuging for activity assay. C-J Flow cytometry to test effects of penfluridol on spleen naïve CD4+T cell differentiation into T cell subsets. C Differentiation from naïve CD4+T to TH1. D Quantification of $\mathbf{C}$. $\mathbf{E}$ Differentiation from naïve CD4+T to TH2. F Quantification of E. G Differentiation from naïve CD4+T to TH17. H Quantification of G. I Differentiation from naïve CD4+T to Treg. $\mathbf{J}$ quantification of $\mathbf{I}$. Experiments were performed for 3 biological replications and one-way analysis of variance and Bonferroni post hoc test were used to test statistical significance of the differences among groups. (NS = not significance, ${ }^{*} p<0.05,{ }^{* *} p<0.01,{ }^{* * *} p<0.001,{ }^{* * *} p<0.0001$ ). PF: penfluridol

and TNFa receptors (Sfig. 6 A-B). We next performed bioinformatics analysis which led to the identification of ASM as a potential target of penfluridol. We undertook several methods to test the association between penfluridol and ASM. Firstly, a DARTS assay was employed to determine the binding of penfluridol and ASM. DARTS results showed elevated protein level of ASM in the presence of penfluridol at the ratio 1:1600 of 
pronase versus protein, indicating that penfluridol bound to ASM and protected ASM from pronase-mediated digestion (Fig. 6A). Secondly, we assessed the ASM activity to test whether the binding of penfluridol to ASM would affect the ASM activity and found that ASM activity was significantly inhibited by penfluridol (Fig. 6B). As ASM activity could be enhanced by inflammatory cytokines, such as TNF $\alpha$, we further investigated whether TNF $\alpha$ elevated ASM activity could be suppressed by penfluridol. Results showed ASM activity was significantly enhanced by TNF $\alpha$, which were obviously inhibited by penfluridol in a dose-dependent manner (Fig. 6B). However, western blotting analysis revealed that penfluridol did not affect the expression of ASM in the presence or absence of TNF $\alpha$ stimulation (Sfig. 7 A-B). Collectively, penfluridol could inhibit the ASM activity through direct binding to it.

Given that ASM was the target of penfluridol and penfluridol could inhibit the production of inflammatory cytokines induced by TNF $\alpha$, we set to investigate whether the inhibitory effects of penfluridol on TNF $\alpha$-induced cytokine production depend on ASM. Thus, effects of penfluridol on mRNA expression and cytokine secretion level were detected after knocking down ASM using siRNA (Sfig. 8A). After ASM knockdown, the inhibitory effect of penfluridol on TNF $\alpha$-induced mRNA expressions and secretions of inflammatory cytokines, including IL-1 $\beta$, IL-6, and NOS-2, were largely lost, suggesting the inhibitory effects of penfluridol on TNF $\alpha$-induced NF- $\mathrm{kB}$ activity was at least partially dependent on ASM (Sfig. 8 B-E). To sum up, ASM was a target of penfluridol, and penfluridol exerted an anti-inflammatory effect through binding to ASM and inhibiting the activity of ASM.

Previously published data reported that ASM played an important role in regulating the differentiation of naive $\mathrm{CD} 4+\mathrm{T}$ cell into $\mathrm{T}$ cell subsets $[36,37]$. Since ASM was identified as a target of penfluridol, we tested whether penfluridol affected naive splenic $\mathrm{CD} 4+\mathrm{T}$ cell differentiation in vitro. Results showed penfluridol inhibited naive $\mathrm{CD} 4+\mathrm{T}$ cell differentiation into $\mathrm{TH} 1$ and TH17 dose-dependently while penfluridol did not affect the differentiation of $\mathrm{CD} 4+\mathrm{T}$ cells to $\mathrm{TH} 2$ and Treg (Fig. 6C-J).

\section{Discussion}

Penfluridol was isolated as a small-molecule drug that could inhibit TNF $\alpha$-induced NF- $\mathrm{KB}$ activation and production of inflammatory cytokines. Further study found ASM was the target of penfluridol and demonstrated penfluridol could inhibit naive CD4+T cell differentiation to TH1 and TH17. Previously published reviews have suggested that ASM could mediate activation of NF- $\mathrm{KB}$ stimulated by TNF $\alpha$ [38-40]. Specifically, after TNF $\alpha$ binding to TNF $\alpha$ receptor 1 (55 $\mathrm{kDa}$ ), phosphatidylcholine-specific phospholipase $\mathrm{C}$ is activated, which hydrolyzes phosphatidyicholine to produce 1,2-diacylglycerol, the well-known second messenger which is a activator of the ASM, and after the activation of ASM, it can regulate the activation of NF- $\mathrm{KB}$ through the production of ceramide which might affect proteolytic degeneration of ІкB $\alpha$. Thus, the NF- $\mathrm{kB}$ signaling pathway is activated, and a positive feedback loop is established to induce the production of the inflammatory cytokines TNF $\alpha$, IL-1 $\beta$, IL-6, and IL-23, which on the one hand promote the production of more inflammatory cytokines and on the other hand affect $\mathrm{T}$ cell differentiation and macrophage polarization. Accordingly, inhibiting the TNF $\alpha$-stimulated NF- $\mathrm{kB}$ signaling pathway exerts antiinflammatory effects and can inhibit the differentiation of naïve $\mathrm{CD} 4+\mathrm{T}$ cells.

Inflammatory cytokines are released by a wide range of immune cells, such as macrophages and $\mathrm{T}$ cells, and play a role in regulating both naive $\mathrm{CD} 4+\mathrm{T}$ cell differentiation and macrophage polarization. For instance, IL-1 $\beta$ and IL- 6 are involved in the differentiation of naive CD4+T cell to TH17, and TNF $\alpha$, IL-1, and IL-6 can promote the macrophage polarization to M1 [35, 41,42 ]. In addition, TNF $\alpha$, IL- $1 \beta$, and IL- 6 secreted by macrophages were reported to induce the expressions of the transcription factor T-bet and RORyt in T cells, leading to the differentiation from naïve $\mathrm{CD} 4+\mathrm{T}$ cells to TH1 and TH17 cells [43].

Penfluridol was reported as a functional inhibitor of ASM by Kornhuber in 2011 [44, 45]. To investigate the inhibitory effects of penfluridol on ASM, we endeavored to elucidate whether penfluridol bound to ASM by bioinformatics analysis, and results suggested their binding, which was further confirmed by DARTS assay. These results provide evidence that penfluridol might be used to treat conditions associated with increased ASM/ceramide levels, like Alzheimer's disease, major depression, radiation- and chemotherapyinduced apoptosis, and endotoxic shock syndrome. Nevertheless, longer-term use of penfluridol may cause off target effects as it has been reported by recent studies that penfluridol could induce apoptosis, suppress Akt and mitogen-activated protein kinase activities, and augment intracellular ROS levels [46-48].

Several limitations have been also noted for this study. Firstly, more dosages and frequencies, including the lower dose group $(0.4 \mathrm{mg} / \mathrm{kg})$, for the treatment of 
arthritis need further investigations for the follow-up preclinical studies. Secondly, for the in vivo study, we only adopted firstline drugs, MTX and 5-ASA, as the positive controls, considering they are all small-molecule drugs. Whether the treatment effect of penfluridol is comparable to other relevant inhibitors, such as Enbrel and Humira, which inhibit NF- $\mathrm{kB}$ activation as biologics, needs further study. Thirdly, the CD4+T cells isolated from spleen by naive $\mathrm{CD} 4+\mathrm{T}$ isolation kit may only have naive $\mathrm{CD} 4+\mathrm{T}$ cells, whether penfluridol has the same inhibitory effect on memory $\mathrm{CD} 4+\mathrm{T}$ cells as on naïve $\mathrm{CD} 4+\mathrm{T}$ cells also needs further study. Finally, whether ASM is required for the activation of NF- $\mathrm{KB}$ is contradictory. Previous studies reported that TNF $\alpha$ could activate NF- $k B$ by ceramide produced by ASM hydrolyzing sphingomyelin in immune cells [40] whereas Kuno 1994 and Zumbansen 1997 et al. [49, 50] suggested TNF $\alpha$ could activate NF- $\kappa B$ in ASM-deficient Niemann-Pick disease type A fibroblasts and ASM knockout embryonic fibroblasts, suggesting the activation of NF- $k B$ is through the canonical signaling pathway without relying on the ASM. Nevertheless, our study showed the inhibitory effect of penfluridol on cytokine production was lost after knockdown ASM in Raw 264.7 macrophages, suggesting the cytokine production induced by TNF $\alpha$ partially relied on ASM. This may be explained by the fact that different cell types were used in these studies and this paradoxical controversy warrants further studies.

\section{Conclusions}

To sum up, penfluridol is a newly found drug that can inhibit TNF $\alpha$-induced NF- $\mathrm{KB}$ activity by targeting ASM that penfluridol binds to, thus reduces ASM activity, which not only provides further understanding of the treatment mechanism for penfluridol but also broadens new possible applications of penfluridol to autoimmune diseases and elevated ASM activity-related diseases, in addition to the well-known role in treating chronic schizophrenia.

\footnotetext{
Abbreviations

TNFa: Tumor necrosis factor alpha; RA: Rheumatoid arthritis; IBD: Inflammatory bowel disease; TNFi: TNFa inhibitors; ACR50: American College of Rheumatology standard; hTNF-TG: Human TNFa transgenic; WT: Wild-type; DMEM: Dulbec co's modification of Eagle's medium; FBS: Fetal bovine serum; TGF: Transforming growth factor; DSS: Ddextran sulphate sodium salt; TNBS: 2,4,6-Trinitrobenzenesulfonic acid solution; ELISA: Enzyme-linked immunosorbent assay; qRT-PCR: Real-time quantitative polymerase chain reaction; IL-1 $\beta$ : Interleukin-1 beta; NOS2: Nitric Oxide Synthase 2; BMDM: Bone marrow-derived macrophages; M-CSF: Macrophage colony-stimulating factor; RANKL: Receptor activating NF-KB ligand; CIA: Collagen-induced arthritis; 5-ASA: 5-Aminosalicylic acid; MTX: Methotrexate; UC: Ulcerative colitis; CD: Crohn's disease.
}

\section{Supplementary Information}

The online version contains supplementary material available at https://doi. org/10.1186/s13075-021-02713-6.

\section{Additional file 1.}

Additional file 2: Sfig. 1 Quantification of western blotting. Sfig. 2 Penfluridol decreases cytokine expression and secretion induced by TNFa in Raw 264.7 cells. Sfig. 3 Penfluridol decreases cytokine expression and secretion in hTNF-TG BMDMs. Sfig. 4 Penfluridol inhibits osteoclastogenesis and macrophage polarization. Sfig. $\mathbf{5}$ mRNA expression levels of CXCL10 and MCP-1 are decreased in penfluridol treated mouse models. Sfig. 6 Penfluridol does not affect binding of TNFa to the receptors. Sfig. 7 Effect of Penfluridol on ASM expression detected by western Blotting. Sfig. 8 Effect of penfluridol on inflammatory cytokine production after knock down ASM. Supplementary Methods.

\section{Acknowledgements}

None.

\section{Authors' contributions}

YC and RL designed and performed experiments, collected and analyzed data, and wrote and revised the paper. WF and YC participated in the project discussions, design of the experiments, analysis of the data, and revision of the paper. AH, LZ, and CZ assisted in the experiments and collected and analyzed data, and assisted in editing the manuscript. C $\mathrm{L}$ designed and supervised this study, analyzed the data, and wrote and edited the manuscript. All author(s) read and approved the final manuscript.

\section{Funding}

This work was funded partly by NIH research grants R01AR062207, R01AR061484, R01AR076900, R01NS103931, a DOD research grant W81XWH-16-1-0482, and CSC 201606240021.

Availability of data and materials

All data are presented in tables or can be found in supplementary documents.

\section{Declarations}

\section{Ethics approval and consent to participate}

All animal experiments were performed in accordance with protocols approved by the Institutional Animal Care and use Committee of New York University School of Medicine.

\section{Consent for publication}

Not applicable.

\section{Competing interests}

The authors declare that they have no competing interests.

\section{Author details}

${ }^{1}$ Department of Orthopaedic Surgery, New York University Grossman School of Medicine, Rm 1608, HJD, 301 East 17th Street, New York, NY 10003, USA. ${ }^{2}$ Department of Rheumatology and Immunology, West China Hospital, Sichuan University, Chengdu 610000, China. ${ }^{3}$ Department of Cell Biology, New York University Grossman School of Medicine, New York, NY 10016, USA.

Received: 20 August 2021 Accepted: 23 December 2021

Published online: 19 January 2022

\section{References}

1. Aggarwal BB, Gupta SC, Kim JH. Historical perspectives on tumor necrosis factor and its superfamily: 25 years later, a golden journey. Blood. 2012;119(3):651-65. 
2. Bacher S, Schmitz ML. The NF-kappaB pathway as a potential target for autoimmune disease therapy. Curr Pharm Des. 2004;10(23):2827-37.

3. Udalova I, Monaco C, Nanchahal J, Feldmann M. Anti-TNF therapy. Microbiol Spectr. 2016:4:4.

4. Chen Y, Sun J, Yang Y, Huang Y, Liu G. Malignancy risk of anti-tumor necrosis factor alpha blockers: an overview of systematic reviews and meta-analyses. Clin Rheumatol. 2016;35(1):1-18.

5. Smolen JS, Weinblatt ME. When patients with rheumatoid arthritis fail tumour necrosis factor inhibitors: what is the next step? Ann Rheum Dis. 2008;67(11):1497-8.

6. Hyrich KL, Lunt M, Watson KD, Symmons DP, Silman AJ. Outcomes after switching from one anti-tumor necrosis factor alpha agent to a second anti-tumor necrosis factor alpha agent in patients with rheumatoid arthritis: results from a large UK national cohort study. Arthritis Rheum. 2007:56(1):13-20.

7. Nurmohamed MT, Dijkmans BA. Efficacy, tolerability and cost effectiveness of disease-modifying antirheumatic drugs and biologic agents in rheumatoid arthritis. Drugs. 2005;65(5):661-94.

8. Gu T, Mutebi A, Stolshek BS, Tan H. Cost of biologic treatment persistence or switching in rheumatoid arthritis. Am J Manag Care. 2018;24(8 Spec):Sp338-sp345.

9. Chong CR, Sullivan DJ Jr. New uses for old drugs. Nature. 2007:448(7154):645-6.

10. Liu R, Chen Y, Fu W, Wang S, Cui Y, Zhao X, et al. Fexofenadine inhibits TNF signaling through targeting to cytosolic phospholipase A2 and is therapeutic against inflammatory arthritis; 2019.

11. Janssen PA, Niemegeers CJ, Schellekens KH, Lenaerts FM, Verbruggen FJ, Van Nueten JM, et al. The pharmacology of penfluridol (R 16341) a new potent and orally long-acting neuroleptic drug. Eur J Pharmacol. 1970;11(2):139-54.

12. Shapiro AK, Shapiro E, Eisenkraft GJ. Treatment of Tourette disorder with penfluridol. Compr Psychiatry. 1983;24(4):327-31.

13. Lee HK, Chung PM, Wang SC. Mechanism of antiemetic action of penfluridol in the dog. Eur J Pharmacol. 1978:53(1):29-38.

14. van Praag HM, Schut T, Dols $L$, van Schilfgaarden R. Controlled trial of penfluridol in acute psychosis. Br Med J. 1971;4(5789):710-3.

15. Soares BG, Lima MS. Penfluridol for schizophrenia. Cochr Database Syst Rev. 2006;(2):Cd002923. https://doi.org/10.1002/14651858.CD002923. pub2.

16. Nikvarz N, Vahedian M, Khalili N. Chlorpromazine versus penfluridol for schizophrenia. Cochr Database Syst Rev. 2017:9:Cd011831.

17. Schettini G, Judd AM, MacLeod RM. Penfluridol decreases secretagogueinduced TSH, GH, and LH secretion in vitro: a possible role for calciumcalmodulin. Neuroendocrinology. 1983;37(3):229-34.

18. Bergamo E, Diani E, Bertazzoni U, Romanelli MG. A luciferase functional quantitative assay for measuring NF-kB promoter transactivation mediated by HTLV-1 and HTLV-2 tax proteins. Methods Mol Biol. 2017:1582:79-87.

19. Keffer J, Probert L, Cazlaris H, Georgopoulos S, Kaslaris E, Kioussis D, et al. Transgenic mice expressing human tumour necrosis factor: a predictive genetic model of arthritis. EMBO J. 1991;10(13):4025-31.

20. Li P, Schwarz EM. The TNF-alpha transgenic mouse model of inflammatory arthritis. Springer Semin Immunopathol. 2003;25(1):19-33.

21. Li G, Wu Y, Jia H, Tang L, Huang R, Peng Y, et al. Establishment and evaluation of a transgenic mouse model of arthritis induced by overexpressing human tumor necrosis factor alpha. Biol Open. 2016;5(4):418-23.

22. Zhuang Y, Liu J, Ma P, Bai J, Ding Y, Yang H, et al. Tamarixinin a alleviates joint destruction of rheumatoid arthritis by blockade of MAPK and NFkappaB activation. Front Pharmacol. 2017;8:538.

23. Brand DD, Latham KA, Rosloniec EF. Collagen-induced arthritis. Nat Protoc. 2007;2(5):1269-75

24. Pietrosimone KM, Jin M, Poston B, Liu P. Collagen-induced arthritis: a model for murine autoimmune arthritis. Bio-protocol. 2015;5(20):e1626.

25. Wei F, Zhang Y, Jian J, Mundra JJ, Tian Q, Lin J, et al. PGRN protects against colitis progression in mice in an IL-10 and TNFR2 dependent manner. Sci Rep. 2014;4:7023.

26. Wirtz S, Neufert C, Weigmann B, Neurath MF. Chemically induced mouse models of intestinal inflammation. Nat Protoc. 2007;2(3):541-6.

27. Wang $X$, Sun $Y$, Zhao $Y$, Ding $Y, Z$ hang $X$, Kong $L$, et al. Oroxyloside prevents dextran sulfate sodium-induced experimental colitis in mice by inhibiting NF-kappaB pathway through PPARgamma activation. Biochem Pharmacol. 2016;106:70-81.

28. Xinqiang S, Fei L, Nan L, Yuan L, Fang Y, Hong X, et al. Therapeutic efficacy of experimental rheumatoid arthritis with low-dose methotrexate by increasing partially CD4+CD25+ Treg cells and inducing Th1 to Th2 shift in both cells and cytokines. Biomed Pharmacother. 2010;64(7):463-71.

29. Erben U, Loddenkemper C, Doerfel K, Spieckermann S, Haller D, Heimesaat MM, et al. A guide to histomorphological evaluation of intestinal inflammation in mouse models. Int J Clin Exp Pathol. 2014;7(8):4557-76.

30. Daina A, Michielin O, Zoete V. SwissTargetPrediction: updated data and new features for efficient prediction of protein targets of small molecules. Nucleic Acids Res. 2019:47(W1):W357-w364.

31. Pai MY, Lomenick B, Hwang H, Schiestl R, McBride W, Loo JA, et al. Drug affinity responsive target stability (DARTS) for small-molecule target identification. Methods Mol Biol. 2015;1263:287-98.

32. Lomenick B, Jung G, Wohlschlegel JA, Huang J. Target identification using drug affinity responsive target stability (DARTS). Curr Prot Chem Biol. 2011;3(4):163-80.

33. Sekiya T, Yoshimura A. In vitro Th differentiation protocol. Methods Mol Biol. 2016;1344:183-91.

34. Flaherty S, Reynolds JM. Mouse naive CD4+ T cell isolation and in vitro differentiation into T cell subsets. J Visualized Exper. 2015;(98):52739.

35. Liu YC, Zou XB, Chai YF, Yao YM. Macrophage polarization in inflammatory diseases. Int J Biol Sci. 2014;10(5):520-9.

36. Bai A, Guo Y. Acid sphingomyelinase mediates human CD4(+) T-cell signaling: potential roles in T-cell responses and diseases. Cell Death Dis. 2017:8(7):e2963.

37. Zhou Y, Salker MS, Walker B, Munzer P, Borst O, Gawaz M, et al. Acid sphingomyelinase (ASM) is a negative regulator of regulatory $T$ cell (Treg) development. Cellular Physiol Biochem. 2016;39(3):985-95.

38. Schütze S, Potthoff K, Machleidt T, Berkovic D, Wiegmann K, Krönke M. TNF activates NF-kappa B by phosphatidylcholine-specific phospholipase C-induced "acidic" sphingomyelin breakdown. Cell. 1992;71(5):765-76.

39. Schütze $S$, Machleidt T, Krönke M. The role of diacylglycerol and ceramide in tumor necrosis factor and interleukin-1 signal transduction. J Leukoc Biol. 1994;56(5):533-41.

40. Ballou LR, Laulederkind SJ, Rosloniec EF, Raghow R. Ceramide signalling and the immune response. Biochim Biophys Acta. 1996;1301(3):273-87.

41. Burkett PR. Meyer zu Horste G, Kuchroo VK. Pouring fuel on the fire: Th17 cells, the environment, and autoimmunity. J Clin Invest. 2015;125(6):2211-9.

42. Fang P, Li X, Dai J, Cole L, Camacho JA, Zhang Y, et al. Immune cell subset differentiation and tissue inflammation. J Hematol Oncol. 2018;11(1):97.

43. Wallace $K L$, Zheng LB, Kanazawa Y, Shih DQ. Immunopathology of inflammatory bowel disease. World J Gastroenterol. 2014;20(1):6-21.

44. Kornhuber J, Muehlbacher M, Trapp S, Pechmann S, Friedl A, Reichel M, et al. Identification of novel functional inhibitors of acid sphingomyelinase. PLoS One. 2011;6(8):e23852.

45. Kornhuber J, Tripal P, Reichel M, Terfloth L, Bleich S, Wiltfang J, et al. Identification of new functional inhibitors of acid sphingomyelinase using a structure-property-activity relation model. J Med Chem. 2008;51(2):219-37.

46. Wu SY, Wen YC, Ku CC, Yang YC, Chow JM, Yang SF, et al. Penfluridol triggers cytoprotective autophagy and cellular apoptosis through ROS induction and activation of the PP2A-modulated MAPK pathway in acute myeloid leukemia with different FLT3 statuses. J Biomed Sci. 2019;26(1):63.

47. Hedrick E, Li X, Safe S. Penfluridol represses integrin expression in breast cancer through induction of reactive oxygen species and downregulation of Sp transcription factors. Mol Cancer Ther. 2017;16(1):205-16.

48. Ranjan A, Srivastava SK. Penfluridol suppresses pancreatic tumor growth by autophagy-mediated apoptosis. Sci Rep. 2016;6:26165.

49. Kuno K, Sukegawa K, Ishikawa Y, Orii T, Matsushima K. Acid sphingomyelinase is not essential for the $\mathrm{IL}-1$ and tumor necrosis factor receptor signaling pathway leading to NFkB activation. Int Immunol. 1994;6(8):1269-72.

50. Zumbansen M, Stoffel W. Tumor necrosis factor al pha activates NFkappaB in acid sphingomyelinase-deficient mouse embryonic fibroblasts. J Biol Chem. 1997;272(16):10904-9.

\section{Publisher's Note}

Springer Nature remains neutral with regard to jurisdictional claims in published maps and institutional affiliations. 